\title{
Assessing Safety and Efficacy of Uterine Artery Embolization in Symptomatic Uterine Fibroids
}

\author{
HAZEM I. KORASHI, M.D.; AHMED H. SOLIMAN, M.D. and AHMED EL SHIMY, M.D. \\ The Department of Diagnostic and Interventional Radiology, Faculty of Medicine, Ain Shams University
}

\begin{abstract}
Background: Uterine artery embolization (UAE) is a modality for management of symptomatic uterine leiomyomata. UAE for uterine fibroids in Egypt is a procedure with minimal reported data.
\end{abstract}

Aim of Study: To determine safety and efficacy of UAE in treating uterine leiomyomata in Egyptian population.

Patients and Methods: 45 consecutive patients undergoing UAE for uterine leiomyomata management were questioned for fibroid associated manifestation and symptomatic improvement at predetermined follow-up clinical visits. Data were obtained via verbal questionnaire.

Data collected included early, late procedural related complications and possible gynecological interventions were gathered.

Follow-up imaging by MRI at three and six months was carried out in order to measure reduction in uterine, dominant leiomyomata volume post-UAE.

Results: Technical success was achieved in $100 \%$ of patients. No significant complication took place with no necessary post procedural-related gynecological interventions.

Follow-up duration was 6 months. Heavy menstrual bleeding improved in $91.1 \%$ of patients at 3 months and in $95.6 \%$ at 6 months post treatment. Pressure-related symptoms improved in $84.4 \%$ of patients at 3 months and in $95.6 \%$ at 6 months after treatment. Convenient control of pain was evident in $55.6 \%$ of patients at 3 months and in $73.3 \%$ at 6 months post-UAE. MRI showed highly significant reduction in uterine as well as dominant fibroid volumes $(24.4,31.1 \%$ at 3, 6 months), (33.3, 44.4\% at 3, 6 months) respectively.

Conclusion: Uterine artery embolization is an effective procedure in treatment of symptomatic uterine leiomyomata with reduced post-procedural complications.

Key Words: Uterine artery embolization - Uterine leiomyomata - Fibroid.

\section{Introduction}

UTERINE leiomyomas frequently referred to as fibroids are the most commonly occurring tumor in women of child bearing period [1-4] .

Correspondence to: Dr. Hazem I. Korashi, The Department of Diagnostic and Interventional Radiology,

Faculty of Medicine, Ain Shams University
Though benign, leiomyomata may disrupt the normal life rhythm activities by being a cause for troublesome symptoms as like heavy menstrual bleeding, pelvic pain, pressure-related symptoms and infertility problems [4]

Management of symptomatic uterine leiomyomata have been traditionally done by many treatments ranging from medical treatment up to invasive hysterectomy [5-8] . Patients' desire to keep their uterus have created an urgent need for the development of uterus sparing alternatives as laparoscopic and hysteroscopic myomectomies [6,8]

Presence of multiple fibroids with variable size, location adding to associated pain, high postoperative morbidity as well as symptoms recurrence ( $25 \%$ of patients] have made myomectomy not feasible in all circumstances $[2,10]$.

UAE was proposed in 1995 as an alternative uterine -sparing technique for treating symptomatic leiomyomata [9]. Subsequent research has proven its efficacy in the short, mid-term and long term with lower complication rate and reduced hospital stay [10-15]

\section{Patients and Methods}

From March 2016 to March 2017, in interventional Radiology unit, Ain Shams University, we recruited 45 female patients with uterine fibroids. After obtaining consents from patients, and institutional ethical committee approval, all women were first assessed clinically for their most annoying symptom/s; the symptoms were grouped as follows:

1- 32 patients ( $71.1 \%$ of patients) having heavy menstrual bleeding as a chief complaint, nominated as bleeding associated with prolongation in time and/or increased amount of the usual menstrual periods relative to the patient's prior experience or resulting in severe anemia. 
2- 8 patients ( $17.7 \%$ of patients) with their main complaint pressure symptoms related to the mass effect of the fibroid.

3- 4 patients ( $8.9 \%$ of patients) presented with infertility attributable to the fibroid (no other defined cause for infertility) and refusing surgical intervention.

4- One patient ( $2.2 \%$ of patients) her main complaint was pain.

The exclusion criteria were patients who are pregnant; patients presented with pedunculated sub-mucosal or cervical leiomyomas; those with renal dysfunction, contrast hypersensitivity and those having bleeding tendency.

All patients had pelvic magnetic resonance imaging (MRI) done using post-contrast enhancement series to unify the measurements of the lesions preand post-procedure in the three orthogonal dimensions aiming at calculating their volumes, defining their location and assessing other pathologies.

The volumes of the uterus and the dominant leiomyomata were calculated in $\mathrm{cm}^{3}$ pre-procedural as a base line and at $3 \& 6$ months post-procedural using the formula for a simplified prolate ellipse (L X W X D X .542) [17].

The procedures were done under local anesthesia with fluoroscopic control using monoplane Toshiba unit with a 5F sheath (TERUMO) and a $5 \mathrm{~F}$ Cobra 2 catheter (Cordis) with a $0.35 \mathrm{~F}$ hydrophilic guide wire (TERUMO).

Selective catheterization of the contralateral (left) uterine artery was implemented first followed by formation of a Waltman loop (which is a longreversed loop obtained with the cobra catheter) and then withdrawal of the catheter to select the ipsilateral (right) uterine artery.

The embolization of both uterine arteries was done after reaching the transverse portion of both uterine arteries with embosphereparticles (BiosphereMedical) $500-700 \mu \mathrm{m}$ (42.2\% of patients), $700-900 \mu \mathrm{m}$ (22.2\% of patients) up to $900-1200 \mu \mathrm{m}$ (13.3\% of patients) followed by torpedoes of gel foam in 9 patients in order to achieve complete flow cessation (Figs. 1,3). Unilateral UAE embolization was performed in one patient due to technical difficulties as a result of arterial spasm.

The technical end point was stasis in the main uterine artery for not less than 10 cardiac beats.

Analgesics and non-steroidal anti-inflammatory medications were prescribed for postoperative pain if present.
Post-procedure outcomes were evaluated diversely as; technical, physical, psychological and clinical improvement.

Patients' data as regard length of hospital stay, days till return to normal activity, complications were assembled. According to Society of Interventional Radiology (SIR) standards; complications were subdivided into: (1) Major complication that necessitates longer hospital stay than expected or that leaves long-lasting adverse effect. (2) Minor complications that can be self-limiting or solved on a day case admission.

Follow-up of the patients' symptoms were monitored 3 and 6 months after discharge by verbal questionnaire. The bleeding, pressure and pain symptoms improvements were graded as follow: (Significantly worse, 1- Slightly worse; 2- Stationary; 3- Slightly improved; 4- Significantly improved 5). Patient psychological satisfaction at these follow-ups visits was also rated as: [Satisfied (3), neither (2), dissatisfied (1)].

Technical success was evaluated by measurement of reduction in both uterine, dominant leiomyoma volumes as well as need for re-interventions whether repeated UAE or subsequent myomectomies and hysterectomies.

\section{Statistical analysis:}

Descriptive data obtained was the procedure parameters, uterine and leiomyoma volume changes, and questionnaire responses. As baseline parameters, the patient age, most annoying symptom, the uterine and dominant fibroid volumes, sum of leiomyomata, and site of dominant leiomyoma were all gathered. IBM SPSS statistics (V. 22.0, IBM Corp., USA, 2013) was used for data analysis. Data were expressed as Median and Percentiles for quantitative non-parametric measures and both number and percentage for categorized data.

The following tests were done: (1) Comparison between 2 dependent groups for parametric data using Paired $t$-test. (2) Chi-square test to study the association between each 2 variables or comparison between 2 independent groups as regards the categorized data. The probability of error at 0.05 was considered significant, while at 0.01 and 0.001 are highly significant.

\section{Results}

A total of 45 patients with average age 36.5 (25-47 yrs.) were included in our study, presenting with uterine leiomyomata with average number of 5 leiomyomatas per patient (ranged from 1 to 12 
leiomyomatas). Recorded average uterine as well as dominant leiomyoma volumes were 810 (350$\left.1205 \mathrm{~cm}^{3}\right), 347.8\left(90-550 \mathrm{~cm}^{3}\right)$ respectively.

The procedure was technically successful on both uterine arteries in 43 patients. In 2 patients, arterial spasm hindered bilateral uterine artery catheterization, hence unilateral approach was done.

All patients were discharged on the same day of the procedure with 9 (5-24) hours average time of hospital stay. Overnight (>24-hour) admission to the hospital was required in $8.7 \%$ of patients. The average numbers of days until patients return to normal activity post-therapy was 7 (5-11) days.

Minor complications were recorded in only $13.3 \%$ of patients; two having post-procedural fever treated with IM antibiotics for 6 days, another patient presented with localized hematoma managed by sufficient compression for 1 hour. Three other patients complained of persistent severe pain for which night admission was required with administered potent analgesics and non-steroidal antiinflammatory agents. Neither major complication nor need for subsequent gynecologic interventions occurred.

Follow-up verbal questionnaires were acquired from all patients at 3,6 months. No one lost followup visits. Most patients assured symptomatic improvement post procedural at 3 months which continued to 6 months of follow-up.

Among patients presented with bleeding $(n=37)$ $(n=36) 91.1 \%, 95.6 \%$ reported symptomatic improvement at 3,6 M follow-up respectively. No significant symptomatic change between 3,6 months follow-up $(p=0.270)$. Twenty patients suf- fered from pressure symptoms, follow-up at 3 months revealed that $84.4 \%$ of patients had improvement (11.1\% significant, $75.6 \%$ slight), $11.1 \%$ had no change, $4.4 \%$ gave slightly worse response. While at 6 months follow-up 95.6\% reported symptomatic improvement (80\% significant, $15.6 \%$ slight), $4.4 \%$ had no change. A highly significant time improvement between 3,6 months follow-up is notable $(p=<0.001)$.

There were 17 patients complaining of pain, at $3 \mathrm{M}$ follow-up $53.3 \%$ get improved, $35.6 \%$ no change, $8.9 \%$ slightly worse response meanwhile at $6 \mathrm{M}$ follow-up $91.1 \%$ get improved $(35.6 \%$ significant, $53.3 \%$ slight), $8.9 \%$ stationary course of pain. There may be clinical reduction of pain from $3,6 \mathrm{M}$ follow-up visits ( $p=0.084$; tend to be clinically significant). Among four patients presented with infertility, one get pregnant at 6 months follow-up with no significant change between $3,6 \mathrm{M}$ followup $(p=0.332)$.

Patient satisfaction paralleled the symptom change. $71.1 \%$ of patients were satisfied at $3 \mathrm{M}$ follow-up which increased up to $88.9 \%$ at $6 \mathrm{M}$.

Imaging follow-up was obtained in 45 patients at 3,6 months after treatment. The mean uterine volume was reduced by $24.4 \%$ (standard deviation [SD] 0.03989) 3 months after treatment and was further reduced by a mean of $31.1 \%$ (SD 0.02796) by 6 months after treatment. The mean dominant fibroid volume was reduced by $33.3 \%$ (SD 0.09802) after 3 months and by $44.4 \%$ (SD 0.06354 ) after 6 months (Fig. 2). A cumulative reduction in both uterine and dominant leiomyoma mean volume from baseline to 6 months post-UAE is evident with significant interval changes between 3,6 months follow-up $(p<0.001)$.
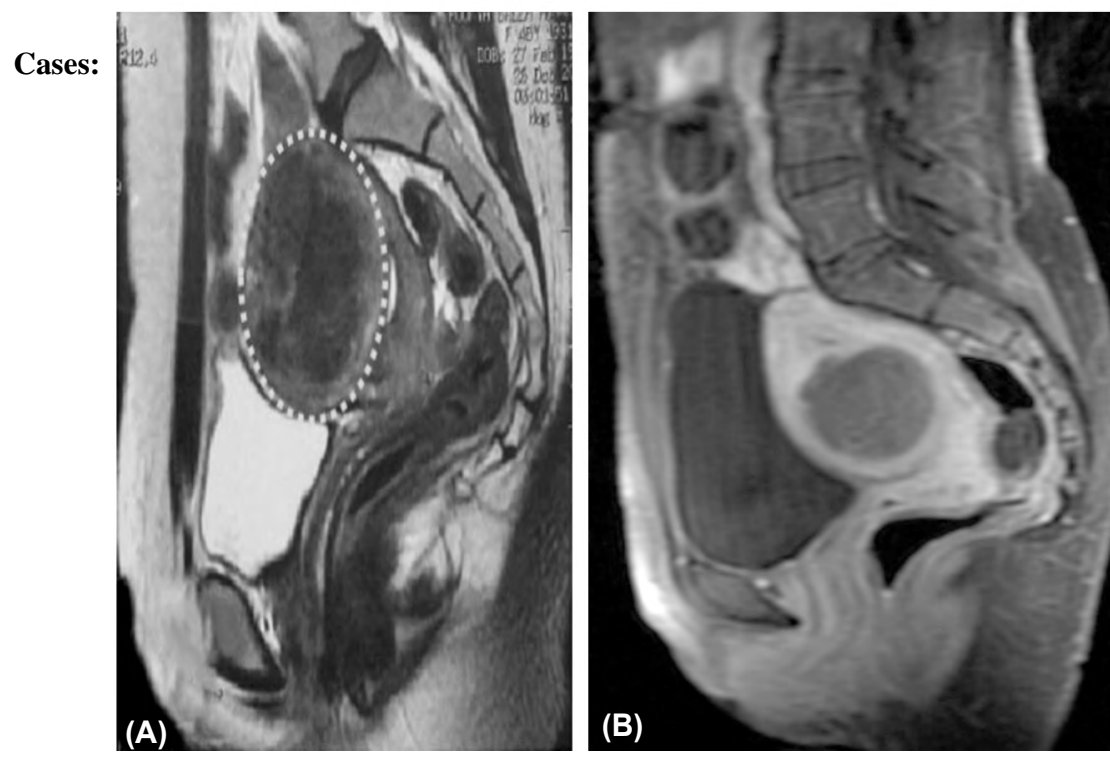

Fig. (1A,B): Sagittal Mю pelvis of 38 years old female patient showing anterior uterine wall myoma pre- (A) and 6 months later (B) Post-embolization which was complaining of meno-metrorrhagia. 

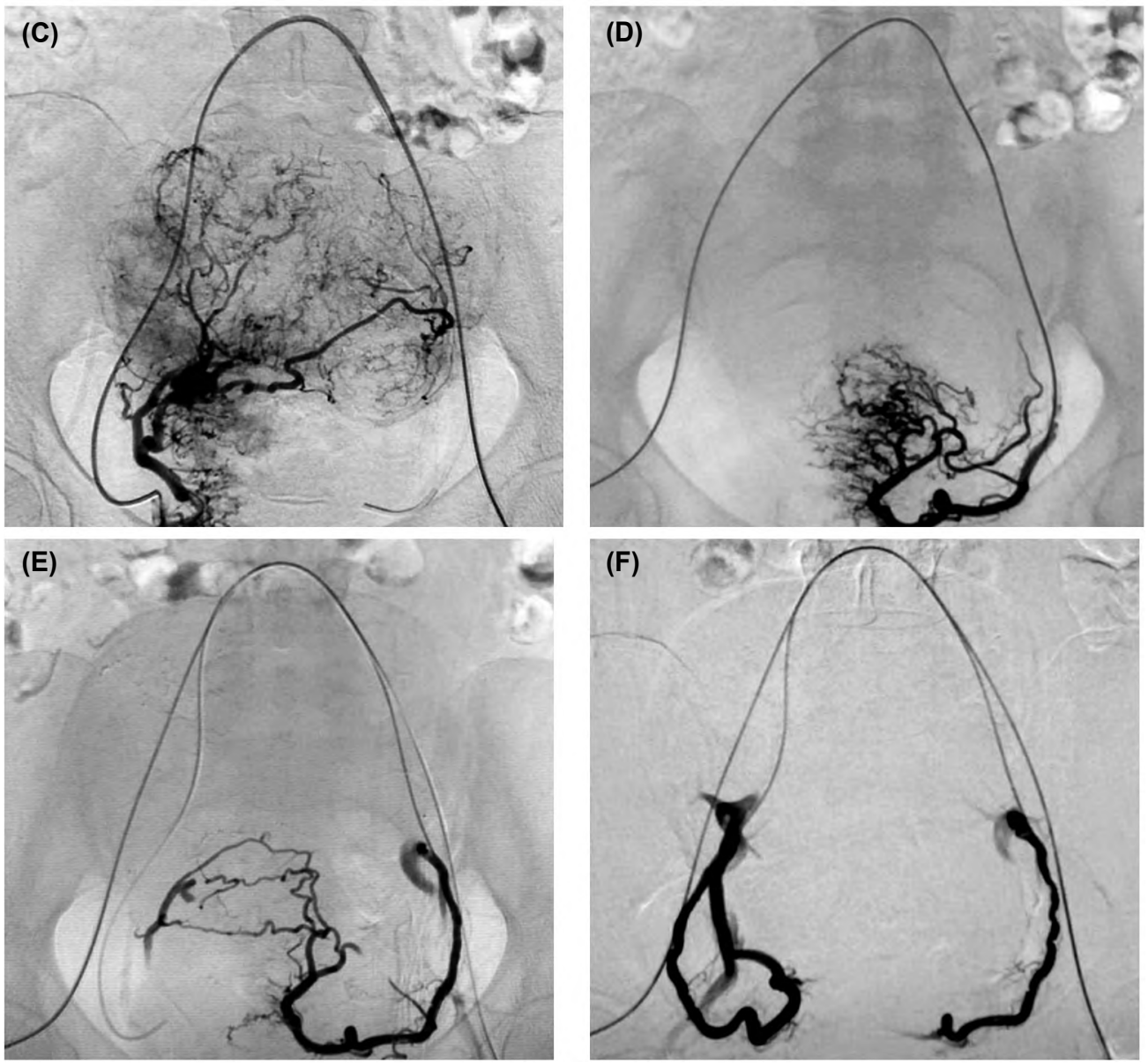

Fig. (1C,D,E,F): Showing: Digital subtraction angiography denoting the fibroid pathological blush during bilateral cross over uterine artery embolization with dominant right uterine artery feeders during the embolization process. (D) DSA control film post embolization with patent main uterine artery bilaterally.

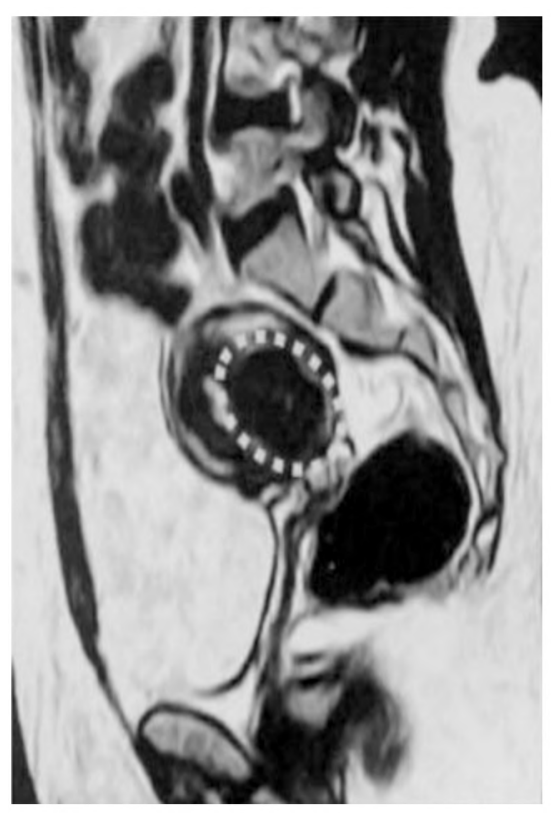

(A)

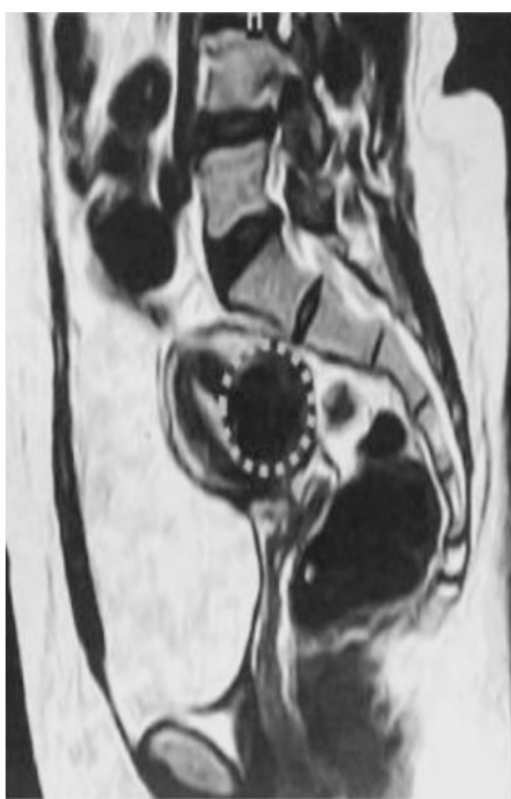

(B)

Fig. (2A,B): Sagittal MRI pelvis of 29 years old female patient showing posterior uterine wall myoma pre (A) measuring $5.4 \times 4 \mathrm{~cm}$ and 3 months later (B) post-embolization measuring $4 \times 3.5 \mathrm{~cm}$ which was complaining of dysmenorrhea. 

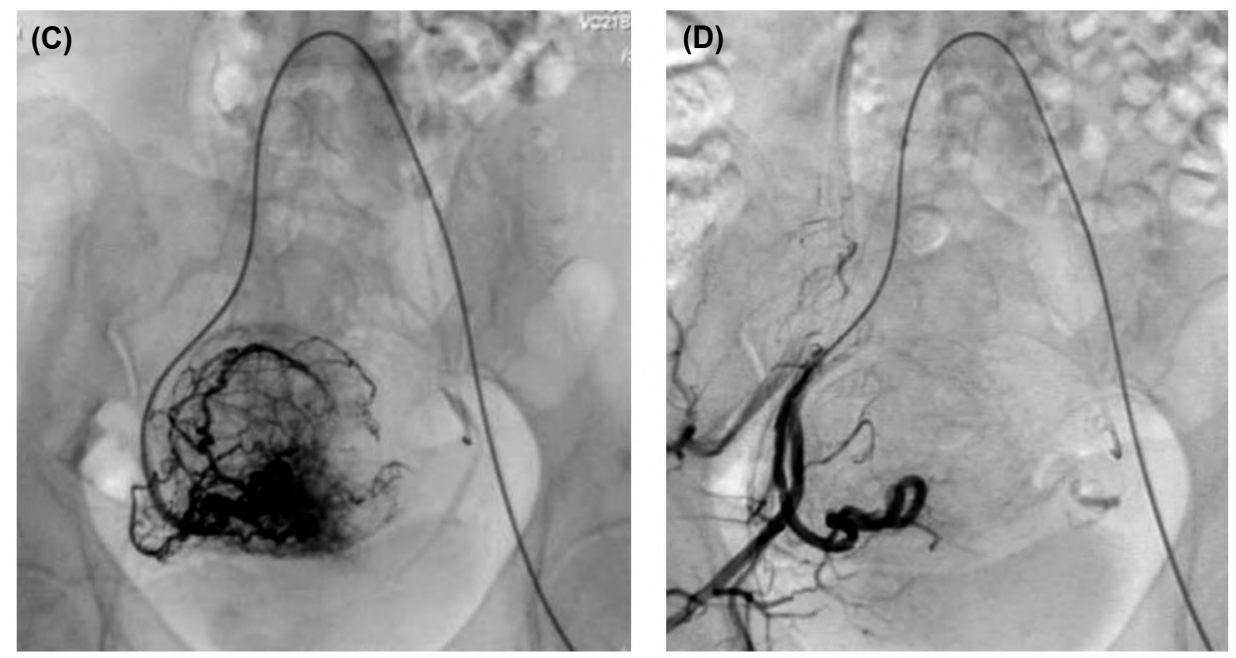

Fig. (2C): Digital subtraction angiography showing right uterine artery catheterization via trans left femoral approach showing fibroid pathological blush, (D) DSA control film post embolization absence of tumoral blush with patent main right uterine artery and iliac divisions
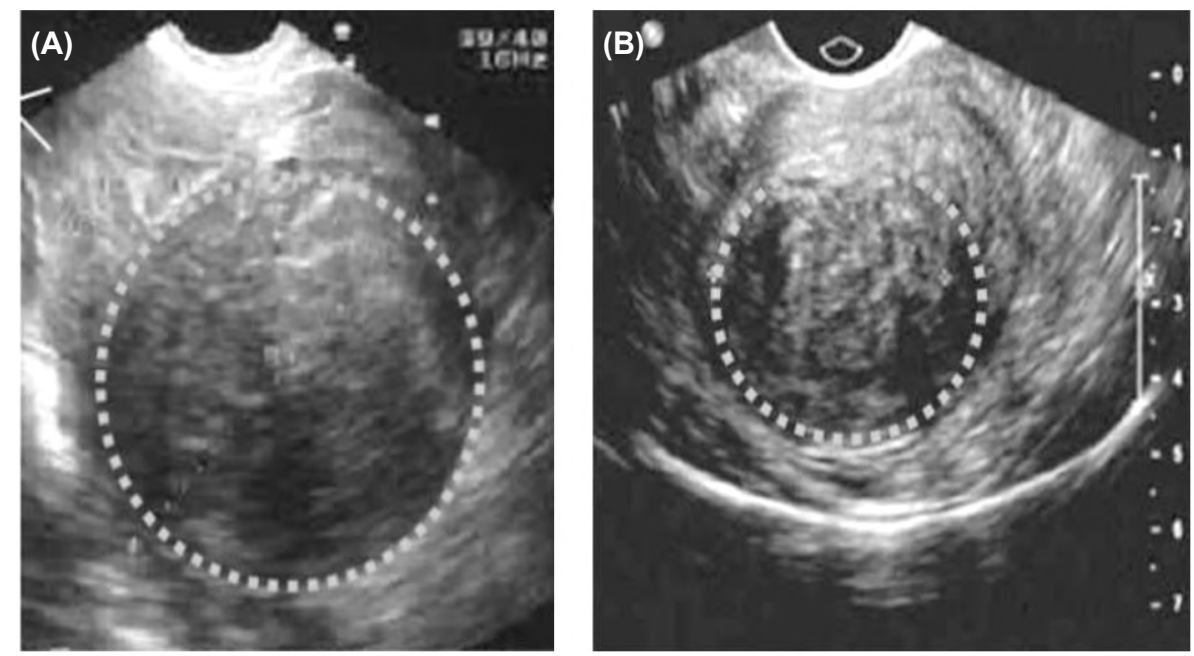

Fig. (3): (A) Transvaginal ultrasonography of 42 years old female patient showing posterior uterine wall myoma pre (A) and 6 months later (B) Post-embolization showing $\pm 60 \%$ volume reduction complaining of dysmenorrhea.
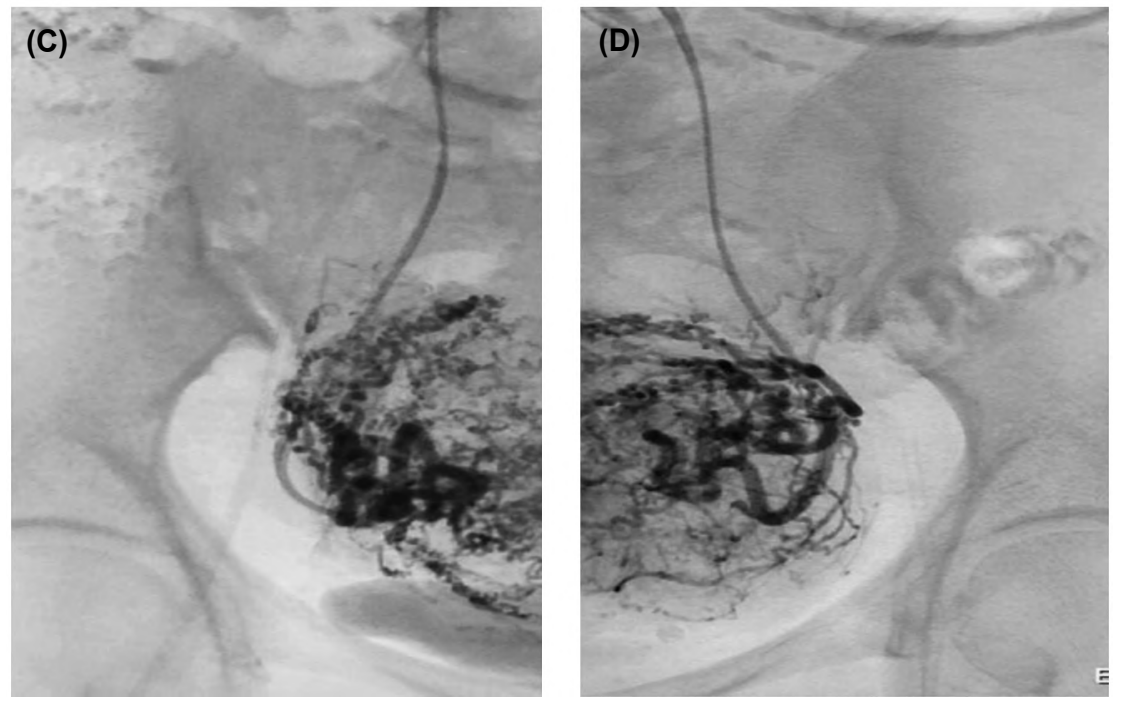

Fig. (3C,D): Digital subtraction angiography showing fibroid tumoral blush from both uterine arteries corresponding to the posterior uterine wall myoma on trans-vaginal ultrasonography. 


\section{Discussion}

Our results with UAE procedure for symptomatic leiomyomata supported those reported in the literature $[\mathbf{8 , 1 5 - 1 8}]$. Heavy menstrual bleeding is controlled in $85-95 \%$ of patients while $70-90 \%$ of patients document improved pressure-related manifestations. In a meta-analysis by Toor et al., rates of symptomatic improvement for less than 1 year follow-up were $87.5 \%$ (84.7-90.0\%) for heavy menstrual bleeding, $86.6 \%(82.2-90.5 \%)$ for pressure-related symptoms, $77.5 \%$ (73.8-80.9\%) for pelvic pain [22]. Our results for menorrhagia, bulk related symptoms are in agreement with those, $92 \%$ of patients reporting improved menstrual bleeding and $85 \%$ of patients with pelvic pressure and other bulk-related symptoms improved at 3 months follow-up post-procedural. These results remained stable with no statistical significance during the course of follow-up.

At $3 \mathrm{M}$ follow-up, $54.4 \%$ of patients presented with pelvic pain report symptomatic improvement which is inferior to that listed in the literature. This may be attributable to consistent complete occlusion of both uterine arteries which may induce relatively severe pain comparable to incomplete embolization as stated by Marsh burn et al., [19]. There was significant time interval cumulative improvement of pain during the period of follow-up to reach $90.9 \%$ at 6 months post-UAE.

Despite normal uncomplicated pregnancy can occur after UAE, it shall be discussed with those willing to retain their infertility or refusing surgical interventions as the two patients included in our study [20]. Among the two patients, one got pregnant at 6 months follow-up. Future prospective studies on larger populations are in need to fully evaluate UAE procedure impact on fertility rates, placenta as well as uterine issues.

Patient satisfaction goes hand in hand with symptomatic improvement, $86.7 \%$ of patient gave satisfactory response at 6 months following the procedure.

Radiological evaluation was done by MRI to precisely measure both uterine and dominant leiomyoma volumes, analyze leiomyomata parameters as regard site, number, enhancement pattern preand post-procedural. Post therapy follow-up detect a cumulative reduction of mean uterine volume by $25 \%, 30 \%$ at 3,6 months respectively and lowering of mean dominant fibroid volume by $34 \%, 44 \%$ after 3,6 months respectively. This goes in agreement with previous literature [8].
Complications were very rare with no detectable post-procedural acute major complications which are near the results reported by meta-analysis of Toor et al., [21]. We had four minor complications; two with fever, local hematoma which were managed by antibiotics, efficient compression; two presented with intolerable pain that necessities overnight admission with potent analgesics. Pelvic pain is the most frequent reported adverse effect of UAE as a result of ischemia, hence the necessity for an appropriate pain management protocol following the procedure. No readmissions had occurred or need for subsequent gynecological interventions till date of publication.

No studies have compared various minimally invasive treatments as ablation therapies with $\mathrm{UAE}$ for symptomatic uterine leiomyomata. Razavi et al., [7,21] concluded that among uterine-sparing therapies for uterine leiomyomata, UAE is minimally invasive safer procedure that achieves better control of menstrual bleeding while myomectomy is more efficacious in improving pressure related symptoms. Both equally control pelvic pain. No known studies have compared impact of UAE and myomectomy on pregnancy outcome, uterus and placenta.

\section{Limitations:}

Based on our study, a multidisciplinary prospective study on a larger population is of paramount importance to specify precisely the clear indications, contraindications and the appropriate candidate for UAE procedure, whom shall benefit from the procedure, who will get worse.

\section{Conflicts of interest:}

We did not receive any fund from any funding body or institute.

\section{Conclusion:}

UAE is an effective procedure for treatment of women with symptomatic uterine leiomyomata with shorter hospital stay, rapid recovery and low rate of major complication. Most patients were satisfied with treatment which goes along with symptomatic improvement.

\section{References}

1- GOODWIN S. and SPIES J.: Uterine Fibroid Embolization N. Engl. J. Med., 361: 690-7, 2009.

2- BRUNEREAU L., HERBRETEAU D., GALLAS S. and COTTIER JP.: Uterine Artery Embolization in the Primary Treatment of Uterine Leiomyomas: Technical Features and Prospective Follow-up with Clinical and Sonographic Examinations in 58 Patients. AJR, 175: 1267-1272, 2000. 
3- BROWNE R., MCCANN J., JOHNSTON C. and MOLLOY M.: Emergency Selective Arterial Embolization for Control of Life-Threatening Hemorrhage from Uterine Fibroids. AJR, 183: 1025-1028, 2004.

4- BEINFELD M., BOSCH J., ISAACSON K. and GAZELLE G.: Cost-Effectiveness of Uterine Artery Embolization and Hysterectomy for Uterine Fibroids. Radiology, 230: 207-213, 2004.

5- EDWARDS R., MOSS J., LUMSDEN M. and WU O.: Uterine-Artery Embolization versus Surgery for Symptomatic Uterine Fibroids. N. Engl. J. Med., 356: 360-70, 2007.

6- DUMOUSSET E., CHABROT P., RABISCHONG B., MAZET N.: Preoperative Uterine Artery Embolization (PUAE) Before Uterine Fibroid Myomectomy. Cardiovasc. Intervent. Radiol., 31: 514-520, 2008.

7- RAZAVI M., HWANG G. and JAHED A.: Abdominal Myomectomy Versus Uterine Fibroid Embolization in the Treatment of Symptomatic Uterine Leiomyomas. American Journal of Roenterology, 180: 1571-1575, 2003.

8- HUTCHINS F., WORTHINGTON-KIRSCH R., and BERKOWITZ R.: Selective Uterine Artery Embolization as Primary Treatment for Symptomatic Leiomyomata Uteri. J. Am. Assoc. Gynecol. Laparosc., 6 (3): 279-284, 1999.

9- SPIES J., ASCHER S. and ROTH A.: Uterine Artery Embolization for Leiomyomata. The American College of Obstetricians and Gynecologists, 98 (1): 29-34, 2001.

10- BUCEK R., PUCHNER S. and LAMMER J.: Mid- and Long-Term Quality-of-Life Assessment in Patients Undergoing Uterine Fibroid Embolization. AJR, 186: 877882, 2006.

11- GOODWIN S., McLUCAS B., LEE M. and CHEN G.: Uterine Artery Embolization for the Treatment of Uterine Leiomyomata Midterm results. JVIR, 10: 1159-1165, 1999.
12- POPOVIC M., BERZACZY D., PUCHNER S. and ZADINA A.: Long-Term Quality of Life Assessment Among Patients Undergoing Uterine Fibroid Embolization. AJR, 193: 267-271, 2009.

13- CHRISTOPHER S. MORRIS.: Update on uterine artery embolization for symptomatic fibroid disease (uterine artery embolization). Abdom Imaging, 33: 104-111, 2008.

14- SHIRLINA D. and SHIRISH S.: Uterine volume: An aid to determine the route and technique of hysterectomy. $\mathrm{J}$. Obstet. Gyneclo. Ind., 54 (1): 68-72, 2004.

15- WALKER W.J., PELAGE J.P.: Uterine artery embolization for symptomatic fibroids: Clinical results in 400 women with imaging follow-up. Br. J. Obstet. Gynaecol., 109: 1262-1272, 2002

16- PRON G., BENNETT J., COMMON A., WALL J., ASCH M. and SNIDERMAN K.: The Ontario Uterine Fibroid Embolization Trial. Part 2. Uterine fibroid reduction and symptom relief after uterine artery embolization forfibroids. Fertil. Steril., 79: 120-127, 2003.

17- WORTHINGTON-KIRSCH R.: Uterine Artery Embolization for Fibroid Disease is Not Experimental. Cardiovasc. Intervent. Radiol., 28: 148-149; 2005.

18-FIROUZNIA K., GHANAATI H., SANAATI M., JALALI A. and SHAKIBA M.: Pregnancy after Uterine Artery Embolization for Symptomatic Fibroids: A Series of 15 Pregnancies. AJR, 192: 1588-1592, 2009.

19- MARSH BURN P., MATTHEWS M., and HURST B.: Uterine Artery Embolization as a Treatment Option for Uterine Myomas. Obstet. Gynecol. Clin. N. Am., 33: 125144, 2006.

20- SPIES J.B.: Recovery after uterine artery embolization: understanding and managing short-term outcomes. J. Vasc. Interv. Radiol., 14: 1219- 22, 2003.

21- TOOR S.S., TAN K.T. and SIMONS M.E.: Clinical failure after uterine artery embolization: evaluation of patient and MR imaging characteristics. J. Vasc. Interv. Radiol., 19: 662-667, 2008 .

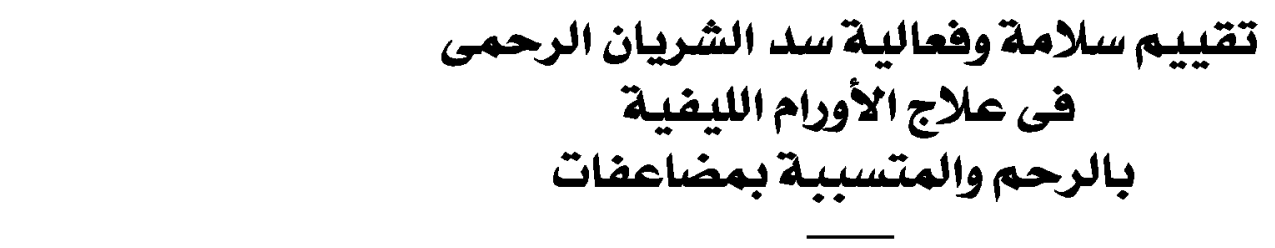

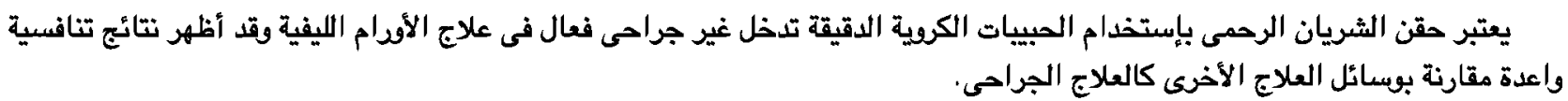
هذه الوبسيلة في العلاج تستحق الأهتمام نظراً لكفايتها في علاج الأفرام الليفية كما أنها توفر نوعية حياة أفضل الرضى.

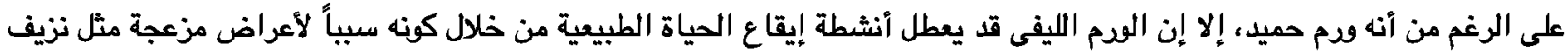
الميض الثقيل وآلام الحوض والأعراض المرتبطة بالضغط على الأعضاء المجاوة ومشياكل العقم. 